PROCEEDINGS OF THE

AMERICAN MATHEMATICAL SOCIETY

Volume 140, Number 10, October 2012, Pages 3607-3622

S 0002-9939(2012)11183-9

Article electronically published on February 6, 2012

\title{
MEASURE AND CUPPING IN THE TURING DEGREES
}

\author{
GEORGE BARMPALIAS AND ANDREW E. M. LEWIS
}

(Communicated by Julia Knight)

\begin{abstract}
We answer a question of Jockusch by showing that the measure of the Turing degrees that satisfy the cupping property is 0 . In fact, every 2-random degree has a strong minimal cover and so fails to satisfy the cupping property.
\end{abstract}

\section{INTRODUCTION}

Upon finding that an order-theoretic property is satisfied by some Turing degrees and not others, there are various approaches that can then be taken in order to further understand the class of degrees for which the property holds. One may attempt to determine the relationship with other order-theoretic properties, or to describe interactions with certain computational properties of sets. Another natural approach is to establish the size of the class of degrees that satisfy the property. This can be done via Baire category or Lebesgue measure 1 For example, Sacks Sac63 showed that the set of minimal degrees is of measure 0 and by Jockusch Joc80 it is also meager. Both of these results say that 'most' degrees are not minimal.

Such an approach can be developed further by using the levels of 'typicalness' that are provided by the theory of genericity and the theory of algorithmic randomness. In the latter we fix a countable family of null sets which we may call effectively null and, identifying sets of natural numbers with their characteristic functions, we say that a set $A \subseteq \mathbb{N}$ is random if it does not belong to any effectively null set. By choosing larger and larger families we can define hierarchies of sets whose levels correspond to higher and higher degrees of randomness or typicalness. The key point here is this: if $P$ is a degree-theoretic property, then there is some degree of randomness which either suffices to ensure satisfaction of $P$ or else suffices to ensure satisfaction of its negation. Similar considerations provide hierarchies of typicalness in terms of Baire category. Given these tools, we can ask the following natural questions.

What properties does the typical Turing degree satisfy and how typical does it have to be in order to satisfy these properties?

In this paper we establish the answer to these questions with respect to the measuretheoretic paradigm, for the case of the cupping property.

Received by the editors January 24, 2011 and, in revised form, March 11, 2011 and April 5, 2011.

2010 Mathematics Subject Classification. Primary 03D28; Secondary 03D10.

The second author was supported by a Royal Society University Research Fellowship.

${ }^{1} \mathrm{By}$ the measure of a set of degrees we mean the measure of the class of sets belonging to them. 
Definition 1.1. A degree $\boldsymbol{a}$ satisfies the cupping property if, for all $\boldsymbol{b}>\boldsymbol{a}$, there exists $\boldsymbol{c}<\boldsymbol{b}$ such that $\boldsymbol{a} \vee \boldsymbol{c}=\boldsymbol{b}$.

Intuitively, the cupping property witnesses a strength of a degree. One of the oldest open questions in the Turing degrees has to do with the related notion of a strong minimal cover. We say that $\boldsymbol{b}$ is a strong minimal cover for $\mathbf{a}$ if the Turing degrees strictly below $\boldsymbol{b}$ are precisely the Turing degrees below and including $\boldsymbol{a}$.

Spector Spe56]: Which degrees have a strong minimal cover?

Notice that if $\boldsymbol{b}$ is a strong minimal cover for $\boldsymbol{a}$, then this degree witnesses the fact that $\boldsymbol{a}$ does not satisfy the cupping property 2 There are a number of ways to make Spector's question more concrete. For example, Yates asked whether every minimal degree has a strong minimal cover 3 Jockusch Joc80 gave a quantitative answer to Spector's question. He showed that every 2-generic set has the cupping property. In particular, the set of degrees with a strong minimal cover is meager (it has no 2-generic members). Moreover this is sharp since by Kumabe Kum00] there is a 1-generic degree with a strong minimal cover.

In a number of talks in the past decade Jockusch asked for a quantitative answer to Spector's question in terms of measure. In this paper we answer Jockusch's question.

Theorem 1.1. Every 2-random degree has a strong minimal cover. Hence the degrees which satisfy the cupping property are of measure 0.

Moreover this result is sharp, in the sense that it does not hold if 2-randomness is replaced with 1-randomness or even weak 2-randomness 4 This follows from the following facts: there is an array non-computable weakly 2-random set [BDN11, and all degrees which are array non-computable satisfy the cupping property [DJS96.

In Section 2 we describe the notation and terminology we shall use in the proof of Theorem 1.1, as well as some background on constructions of strong minimal covers. Section 3 is devoted to the proof of Theorem 1.1. For an introduction to computability theory and the study of the Turing degrees, we refer the reader to Coo04 and Soa87. For an introduction to algorithmic randomness we refer the reader to $\mathrm{Nie09}$ or $\mathrm{DH} 10$.

\section{TERMinOLOGY AND BACKGROUND}

2.1. Cantor space. We let $2^{\omega}$ denote the set of infinite binary sequences and denote the standard Lebesgue measure on $2^{\omega}$ by $\mu$. We let $2^{<\omega}$ denote the set of finite binary strings. A set $\Lambda \subseteq 2^{<\omega}$ is said to be downward closed if, whenever $\tau \in \Lambda$, all initial segments of $\tau$ are in this set. Given any $\Lambda \subseteq 2^{<\omega}$ we denote by $[\Lambda]$ the set of infinite paths through $\Lambda$, i.e. those sets $A$ such that there are an infinite number of initial segments of $A$ in $\Lambda$. If $U \subseteq 2^{<\omega}$ we write [[U]] to denote the set of infinite strings which extend some element of $U$. We let $\chi$ be an effective bijection from $\omega$ to the finite subsets of $2^{<\omega}$ and we write $\lambda$ in order to denote the string of length 0 .

\footnotetext{
${ }^{2}$ Although these two properties are mutually exclusive, it is not known if they are complementary.

${ }^{3}$ The answer to this question is not known. See [Lew09 for a survey.

${ }^{4}$ This notion is an intermediate step between 1-randomness and 2-randomness.
} 
2.2. Machines and trees. We let $W_{e}^{A}$ denote the $e$ th set of finite binary strings which is computably enumerable (c.e.) relative to $A$, according to the underlying universal enumeration of all c.e. sets. We say that $\mathcal{P}$ is a $\Pi_{1}^{0, A}$ class if there exists a downward closed $A$-computable $\Lambda \subseteq 2^{<\omega}$ such that $\mathcal{P}=[\Lambda]$, and we write $\Pi_{1}^{0}$ in order to denote $\Pi_{1}^{0, \emptyset}$.

Generally we shall use the variable $T$ to range over subsets of $2^{<\omega}$ which may not be downward closed. Given any $T \subseteq 2^{<\omega}$ and $\tau, \tau^{\prime} \in T$ we say that $\tau^{\prime}$ is a successor of $\tau$ in $T$ if $\tau \subset \tau^{\prime}$ and there does not exist $\tau^{\prime \prime} \in T$ with $\tau \subset \tau^{\prime \prime} \subset \tau^{\prime}$. A non-empty $T$ is said to be 2-branching if each $\tau \in T$ has precisely two successors in $T$. The strings of level $n$ in $T$ are those strings in $T$ which have precisely $n$ proper initial segments in $T$. We say that $A$ computes $T$ via $\Psi$ if for every $n$ the strings of level $n$ in $T$ are precisely the set $\chi(\Psi(A ; n))$. Since we shall be interested in those Turing functionals which compute 2-branching $T$, we let $\left\{\Psi_{e}\right\}_{e \in \omega}$ be an effective listing of all those Turing functionals $\Psi$ which satisfy the following conditions:

(1) for all $\sigma \in 2^{<\omega}, \Psi(\sigma ; 0)=m$ such that $\chi(m)=\{\lambda\}$;

(2) for $n>0, \Psi(\sigma ; n)$ is defined only if this computation converges in $<|\sigma|$ steps, and $\Psi\left(\sigma ; n^{\prime}\right) \downarrow$ for all $n^{\prime}<n$;

(3) for $n>0$, if $\Psi(\sigma ; n) \downarrow$, then $\chi(\Psi(\sigma ; n))$ is a set of $2^{n}$ pairwise incompatible strings such that precisely two of these strings extend each member of $\chi(\Psi(\sigma ; n-1))$.

A set of strings $T$ is called a perfect tree if every $\sigma \in T$ has at least two incompatible extensions in $T$, and $T$ is called pointed if all paths through $T$ compute $T$. We say that $\sigma \in T$ is a leaf if it does not have any proper extensions in $T$.

2.3. Randomness. We say that a set $P \subseteq 2^{\omega}$ is effectively null if there exists a uniformly c.e. sequence of sets of finite binary strings $\left\{U_{i}\right\}_{i \in \omega}$ such that $\mu\left(U_{i}\right)<$ $2^{-i}$ (where $\mu\left(U_{i}\right)$ means $\left.\mu\left(\left[\left[U_{i}\right]\right]\right)\right)$ and $P \subseteq \bigcap_{i}\left[\left[U_{i}\right]\right]$. Identifying sets with their characteristic functions, we say that a set $A$ is Martin-Löf random [ML66] if it does not belong to any effectively null set. We say that $A$ is Martin-Löf random relative to $B$ if it satisfies this condition when we replace c.e. by c.e. relative to $B$, and we say that $A$ is $n$-random if it is random relative to $\mathbf{0}^{(n-1)}$ (and that a degree is $n$-random if it contains an $n$-random set). Now if a set of reals is of measure 0 , then certainly it is effectively null relative to some oracle.

Martin-Löf randomness is in many respects the standard notion of algorithmic randomness. Other randomness notions may be obtained by varying the level of computability in the above definition. For example, a set is weakly 2-random if it is not a member of any $\Pi_{2}^{0}$ null class.

2.4. Strong minimal covers. Most constructions of strong minimal covers can be seen as extensions of Spector's minimal degree construction. A simple relativization of this method to any degree $\mathbf{a}$ produces a minimal cover for $\mathbf{a}$, i.e. a degree $\mathbf{b}$ such that the interval of degrees $(\mathbf{a}, \mathbf{b})$ is empty. Not every degree has a strong minimal cover. Hence one needs some assumption on a degree $\mathbf{a}$ in order to build a strong minimal cover for it. In Lew07a it was noticed that the following property is sufficient for this purpose.

Definition 2.1. We call $A$ a tree basis if, whenever it computes a perfect tree $T$, it computes a perfect pointed subtree $T^{\prime} \subseteq T$. 
In other words, it was observed that if $A$ is a tree basis, then its degree has a strong minimal cover. In [Lew07b] these ideas were combined with a measuretheoretic construction in order to show that there is a Martin-Löf random degree with a strong minimal cover. Moreover this degree can be chosen to be hyperimmune-free (i.e. every function computed by it is dominated by a computable function). The argument of Section 3 uses these ideas along with new insights in order to show that every 2-random degree has a strong minimal cover. We note that by Martin Mar0s] every 2-random degree is hyperimmune. We do not know if there is a $\Delta_{2}^{0}$ random set with a strong minimal cover.

\section{The Proof of TheOrem 1.1}

In light of the discussion in Section 2.4, to prove Theorem [1.1] it suffices to establish the following lemma:

Lemma 3.1 (Main Lemma). There exists $f \leq_{T} \emptyset^{\prime}$ such that, for any $j, n \in \omega$, $f(j, n)=e$ which satisfies:

- $\mu\left(W_{e}^{\emptyset^{\prime}}\right)<2^{-n}$;

- if $A \notin\left[\left[W_{e}^{\emptyset^{\prime}}\right]\right]$ and $A$ computes a 2-branching tree $T$ via $\Psi_{j}$, then it computes a perfect pointed subtree $T^{\prime} \subseteq T$.

In order to see that Lemma 3.1 suffices to prove Theorem 1.1, define:

$$
U_{k}=\bigcup_{j} W_{f(j, j+k+1)}^{\emptyset^{\prime}} .
$$

Then $\left\{U_{k}\right\}_{k \in \omega}$ is a sequence of sets of finite binary strings which is uniformly c.e. in $\emptyset^{\prime}$ and $\mu\left(U_{k}\right)<2^{-k}$. If $A$ is not a tree basis, then it belongs to $\bigcap_{k}\left[\left[U_{k}\right]\right]$. No 2 -random string can belong to this set since it is effectively null relative to $\emptyset^{\prime}$.

The proof of Lemma 3.1 requires the use of a certain combinatorial lemma. In Section 3.1 we give two simplified versions of it and demonstrate some underlying types of arguments that are needed for the more advanced versions that follow. In Section 3.2 we use these facts in an idealized situation where we prove a version of Lemma 3.1 under additional assumptions. This example contains most ideas of Lew07b, which will be used in the final argument for the proof of Lemma 3.1 . Section 3.3 discusses how we can work without the additional assumptions of Section 3.2, and in Sections 3.4 and 3.5 we obtain the stronger version of the combinatorial lemma that we need in the actual construction along with its measure-theoretic expression. Section 3.6 deals with the construction of the function $f$ of Lemma 3.1 . Finally, in Section 3.7 we give the verification of the construction which concludes the proof.

3.1. Two combinatorial lemmas. By way of an introduction, we prove simplified versions of the combinatorial facts that we need.

Lemma 3.2. Let $X$ and $Y$ be non-empty finite sets such that to each element of $X$ are associated $m$ elements in $Y$ and a 'colour' either 0 or 1 . Then we may colour each element of $Y$ either 0 or 1 in such a way that the proportion of those elements a of $X$ for which there exists at least one associated element of $Y$ that is the same colour as $a$ is at least $m /(m+1)$. 
Proof. We describe a method for colouring the elements of $Y$, which we shall refer to as the 'standard method'.

Stage 0. Define $X_{0}=X$ and $Y_{0}=Y$.

Stage $s+1$. For each element $b$ of $Y_{s}$, each $a \in X_{s}$ and each $i \in\{0,1\}$ we say that giving $b$ colour $i$ aids $a$ if $a$ is associated with $b$ and is coloured $i$. We say that giving $b$ colour $i$ hinders $a$ if $a$ is associated with $b$ and is coloured $1-i$. Choose any $b \in Y_{s}$, choose $i$ so that it aids at least as many elements of $X_{s}$ as it hinders, and give $b$ colour $i$. Define $Y_{s+1}=Y-\{b\}$ and define $X_{s+1}$ to be the elements $a$ of $X$ for which it is not yet the case that there exists at least one associated element of $Y$ which is the same colour as $a$.

In order to see that this procedure does what it is supposed to, we consider two counters $x$ and $y$. Initially $x=y=0$. Whenever we give a colour $i$ to an element $b$ of $Y$ at stage $s+1$ we increase $x$ by the number of elements of $X_{s}$ which this aids, and we increase $y$ by the number of elements that it hinders. Let $x$ and $y$ take their final values. The number of elements $a$ of $X$ for which there exists at least one associated element of $Y$ which is the same colour as $a$ is precisely the final value $x$. For each element $a$ of $X$ for which there is not an associated member of $Y$ of the same colour, we must increase the value $y$ by $m$, in order to rule out correctly colouring each of the $m$ elements of $Y$ with which $a$ is associated. Thus the number of elements $a$ of $X$ for which there does not exist at least one associated element of $Y$ which is the same colour as $a$ is at most $y / m$. Since $x \geq y$ the result follows.

We do not really need the following lemma for our final proof, but we shall use it in the next section in order to introduce techniques that we shall need later.

Lemma 3.3. Let $X$ and $Y$ be non-empty finite sets. Suppose that for each $y<2^{m_{1}}$ and for each $a \in X, f_{y}(a)$ is a subset of $Y$ of size $2^{m_{2}}$. Upon assigning a 'colour', either 0 or 1 , to each element of $X \cup Y$, consider the proportion of $a \in X$ for which the following condition holds: for every $y<2^{m_{1}}$ there exist at least $2^{d}$ elements of $f_{y}(a)$ which are the same colour as $a$. For fixed values of $m_{1}, d$ and $k \in \omega$, if $m_{2}$ is chosen sufficiently large, then, for any colouring of $X$, we can colour the elements of $Y$ so that this proportion is at least $1-2^{-k}$. In fact, there exists a computable function $\pi_{0}$ such that if $m_{2} \geq \pi_{0}\left(m_{1}, k, d\right)$, then this condition will be satisfied (note that this function does not require $|Y|$ as an input).

Proof. Suppose we are given $X$ and $Y$ as in the statement of the lemma and that a colour 0 or 1 has been assigned to each element of $X$. We describe a method for colouring $Y$. We can assume that $m_{2}>m_{1}+2 d$. First we form a set $X^{\prime}$ by replacing each element $a$ of $X$ with $2^{m_{1}+d}$ distinct elements $a_{y, z}$ for $y<2^{m_{1}}$ and $z<2^{d}$. Each of these $2^{m_{1}+d}$ elements that replaces $a$ is given the same colour as $a$. The element $a_{y, z}$ we associate with $2^{m_{2}-d}$ elements of $f_{y}(a)$ in such a way that for $z \neq z^{\prime}$ the set of elements associated with $a_{y, z}$ is disjoint from the set of elements associated with $a_{y, z^{\prime}}$. Then we colour $Y$ according to the standard method for $X^{\prime}$ and $Y$.

Let us say that the colouring is good for $a_{y, z} \in X^{\prime}$ if there exists at least one associated member of $Y$ with the same colour as $a_{y, z}$. The total number of elements in $X^{\prime}$ is $2^{m_{1}+d}|X|$. Since each element of $X^{\prime}$ is associated with $2^{m_{2}-d}$ elements of $Y$, it follows from the proof of Lemma 3.2 that the total number of elements of $X^{\prime}$ for which the colouring is not good is at most $\frac{2^{m_{1}+d}|X|}{2^{m_{2}-d}+1}$. Let us say that the 
colouring is good for $a \in X$ if it is good for every one of the elements of $X^{\prime}$ that replaced it. Note that if the colouring is good for $a \in X$, then for every $y<2^{m_{1}}$ there exist at least $2^{d}$ elements of $f_{y}(a)$ that are the same colour as $a$. The total number of $a \in X$ for which the colouring is not good can be at most the total number of $c \in X^{\prime}$ such that the colouring is not good for $c$. Thus the proportion of $a \in X$ for which the colouring is not good is at most $\frac{2^{m_{1}+d}}{2^{m_{2}-d}+1}$. The proportion of $a \in X$ for which the colouring is good is therefore at least $1-\frac{2^{m_{1}+d}}{2^{m_{2}-d}+1}$. For fixed values of $m_{1}, k$ and $d$, we can effectively find $m$ such that the latter value is greater than $1-2^{-k}$ for all $m_{2} \geq m$.

3.2. A simplified example. We now consider a simplified situation and show the kind of use that can be made of Lemma 3.3

In this simplified situation, we suppose we are given a $\Pi_{1}^{0}$ class $P=[\Lambda]$, where $\Lambda$ is a downward closed and computable set of finite binary strings. We also suppose that for all $A \in P, \Psi_{j}(A)$ is total, and we assume further that $\Psi_{j}$ satisfies a certain convenient condition; for all $A, B$ and for all $m$, if $\chi\left(\Psi_{j}(A ; m)\right) \downarrow$ and $\chi\left(\Psi_{j}(B ; m)\right) \downarrow$, then all strings in these two sets are of the same length. We let $h$ be some computable function such that for all $\sigma \in \Lambda$ of length $h(m), \Psi_{j}(\sigma ; m) \downarrow$.

Now suppose that, for whatever reason, we are given $n \in \omega$ and we wish to form a new $\Pi_{1}^{0}$ class $P^{\prime} \subseteq P$ by removing at most $2^{-n}$ measure from $P$, so that this new class satisfies:

$\mathcal{Q}$ : if $A \in P^{\prime}$ computes a 2-branching $T$ via $\Psi_{j}$, then $A$ computes some 2branching $T^{\prime} \subseteq T$ such that every $B \in\left[T^{\prime}\right]$ computes $A$.

In order to ensure that every $A \in P^{\prime}$ satisfies requirement $\mathcal{Q}$ we shall construct $\Phi$ and $\Xi$ such that if $A \in P^{\prime}$ computes $T$ via $\Psi_{j}$, then $A$ computes a 2-branching $T^{\prime} \subseteq T$ via $\Xi$ and for every $B \in\left[T^{\prime}\right]$ we have $\Phi(B)=A$. We let $\kappa$ be a computable function such that $\sum_{x>0} 2^{-\kappa(x)}<2^{-n}$. We shall describe a computable process such that at each step in the process various clopen sets of strings are removed from $P$. At step $x \geq 1$ we shall remove measure at most $2^{-\kappa(x)}$ from $P$, so that ultimately we can define $P^{\prime}$ to be the set of strings in $P$ which are not removed at any step of the process. The point of this process is to define $\Xi$ and $\Phi$, and the strings we remove from $P$ are those strings on which we are not able to define these values in an appropriate way. At each step $x$ in the process we shall look to define the strings of level $x$ in $T^{\prime}$ which is computed via $\Xi$, and for each string $\tau$ of level $x$ in $T^{\prime}$ we shall look to define $\Phi(\tau ; x-1)$.

First we define $\Xi(\lambda ; 0)=\chi^{-1}(\{\lambda\})$. At step 1 , in order to define $\Phi$ on argument 0 and $\Xi$ on argument 1 , we choose some large $m$ and then, for the various $\sigma \in \Lambda$ of length $h(m)$, we enumerate axioms of the form $\Phi(\tau ; 0)=i \in\{0,1\}$ for $\tau$ in $\chi\left(\Psi_{j}(\sigma ; m)\right)$. We wish to ensure that the proportion of the $\sigma \in \Lambda$ of length $h(m)$ for which there exist at least two corresponding $\tau$ (i.e. $\tau \in \chi\left(\Psi_{j}(\sigma ; m)\right)$ ) for which we enumerate the axiom $\Phi(\tau ; 0)=\sigma(0)$ is greater than $1-2^{-\kappa(1)}$. The hope is that we can achieve this if we choose an $m$ that is sufficiently large. Lemma 3.3 tells us precisely that we can do this. In the statement of the lemma, put $m_{1}=0, d=$ $1, k=\kappa(1)$ and $m_{2}=m$. Think of $X$ as the set of strings $\sigma \in \Lambda$ of length $h(m)$. For each $\sigma \in X$ think of $f_{0}(\sigma)$ as being $\chi\left(\Psi_{j}(\sigma ; m)\right)$. Think of $Y$ as the union of all sets of strings $\chi\left(\Psi_{j}(\sigma ; m)\right)$ such that $\sigma$ is in $X$. The colour given to an element of $X$ should be thought of as the value $\sigma(0)$. Finally, the colour given to elements of $Y$ corresponds to the axioms we enumerate for $\Phi$. Thus we can enumerate the 
axioms for $\Phi$ in such a way that the measure of those strings which extend some $\sigma$ of length $h(m)$ in $\Lambda$, and for which there do not exist at least two corresponding $\tau$ for which we enumerate the axiom $\Phi(\tau ; 0)=\sigma(0)$ is less than $2^{-\kappa(1)}$. At this point we remove all strings extending such a $\sigma$ from $P$ and in doing so we remove measure at most $2^{-\kappa(1)}$. Let $\Pi_{1}$ be the set of strings in $\Lambda$ of length $h(m)$ which remain. For each $\sigma$ in $\Pi_{1}$ we can define $\Xi(\sigma ; 1)$ to be (the code for) a set of two strings in $\chi\left(\Psi_{j}(\sigma ; m)\right)$, each of which correctly computes $\sigma(0)$ via $\Phi$. For all those strings $\sigma$ of length $h(m)$ that remain in $P^{\prime}$ we have successfully defined $T^{\prime}$ which $\sigma$ computes via $\Xi$ up to level 1 . For all strings $\tau$ of level 1 in $T^{\prime}, \Phi(\tau)$ correctly computes $\sigma$ on argument 0 .

We then proceed to define $\Phi$ on argument $1, \Xi$ on argument 2 and $\Pi_{2}$. We choose some large $m^{\prime}$ and then, for the $\sigma \in \Lambda$ of length $h\left(m^{\prime}\right)$ that extend a string in $\Pi_{1}$, we enumerate axioms of the form $\Phi(\tau ; 1)=i \in\{0,1\}$ for $\tau$ in $\chi\left(\Psi_{j}\left(\sigma ; m^{\prime}\right)\right)$. Now we consider the proportion of the $\sigma \in \Lambda$ of length $h\left(m^{\prime}\right)$ that extend a string in $\Pi_{1}$ such that, for each string $\tau$ in $\chi(\Xi(\sigma ; 1))$, there exist at least two strings $\tau^{\prime} \supset \tau$ in $\chi\left(\Psi_{j}\left(\sigma ; m^{\prime}\right)\right)$ for which we enumerate the axiom $\Phi\left(\tau^{\prime} ; 1\right)=\sigma(1)$. We wish to ensure that this proportion is greater than $1-2^{-\kappa(2)}$. Once again, Lemma 3.3 tells us precisely that we can do this. Put $m_{1}=1, d=1, k=\kappa(2)$ and $m_{2}=m^{\prime}-m$. Think of $X$ as the set of strings $\sigma \in \Lambda$ of length $h\left(m^{\prime}\right)$ which extend a string in $\Pi_{1}$. For each $y<2$ and each $\sigma \in X$ think of $f_{y}(\sigma)$ as the set of strings in $\chi\left(\Psi_{j}\left(\sigma ; m^{\prime}\right)\right)$ which extend the $y^{\text {th }}$ element of $\chi(\Xi(\sigma ; 1))$ (ordered from left to right, say). Think of $Y$ as the union of all these sets $f_{y}(\sigma)$ such that $\sigma \in X$ and $y<2$. The colour given to an element $\sigma$ of $X$ now corresponds to the value $\sigma(1)$. Once we have enumerated the axioms for $\Phi$ on argument 1 , let $\Pi_{2}$ be the set of those $\sigma$ of length $h\left(m^{\prime}\right)$ such that, for each string $\tau$ in $\chi(\Xi(\sigma ; 1))$ there exist at least two strings $\tau^{\prime} \supset \tau$ in $\chi\left(\Psi_{j}\left(\sigma ; m^{\prime}\right)\right)$ for which we enumerate the axiom $\Phi\left(\tau^{\prime} ; 1\right)=\sigma(1)$. We can then remove all those elements of $P$ which do not extend a string in $\Pi_{2}$ and the measure of the elements removed will be at most $2^{-\kappa(2)}$. For the strings $\sigma$ in $\Pi_{2}$ we can define $\Xi(\sigma ; 2)$ to be (the code for) a set of four pairwise incompatible strings in $\chi\left(\Psi_{j}\left(\sigma ; m^{\prime}\right)\right)$ each of which correctly computes $\sigma(1)$ via $\Phi$, and such that precisely two of these strings extend each element of $\chi(\Xi(\sigma ; 1))$. Then we proceed by induction in the obvious way.

There are two ways in which the situation we are presented with as we look to prove Theorem [1.1 is more complicated than the simplified situation we just considered. Firstly, we do not actually get to work within a $\Pi_{1}^{0}$ class $P$ such that $\Psi_{j}$ is total for all elements of $P$. Instead we have to work with all sets, and we do not know the measure of the class of sets on which $\Psi_{j}$ is total. Secondly, the functional $\Psi_{j}$ may not behave as tidily as we assumed it did in the previous discussion: it will not necessarily be the case that for all $A, B$ and for all $m$, if $\chi\left(\Psi_{j}(A ; m)\right) \downarrow$ and $\chi\left(\Psi_{j}(B ; m)\right) \downarrow$, then all strings in these two sets are of the same length.

In fact the second of these apparent problems is very easily dealt with, so we address this first. The following lemma provides the necessary tool.

Definition 3.1. For fixed values $\sigma \in 2^{<\omega}$ and $j \in \omega$, we define:

$$
T_{j}(\sigma)=\bigcup\left\{\chi\left(\Psi_{j}(\sigma ; m)\right): \Psi_{j}(\sigma ; m) \downarrow\right\} .
$$

Note that, according to the conventions established in Section 2.2, $T_{j}(\sigma)$ will be finite. A string $\tau$ is compatible with $T \subseteq 2^{<\omega}$ if it has an extension in $T$. 
Lemma 3.4. Suppose that $\mathcal{P}=[\Lambda]$, that $\Lambda$ is downward closed (but not necessarily computable) and that $\Psi_{j}(A)$ is total for all $A \in P$. Let $X$ be a finite and prefix-free set of strings in $\Lambda$ and let $l_{0}=\max \{|\sigma|: \sigma \in X\}$. For any $m$ there exists $l>l_{0}$ and $l^{\prime}$ such that, for every string $\sigma_{1}$ in $\Lambda$ of length $l$ which extends some element $\sigma_{0}$ of $X$, and for each leaf $\tau$ of $T_{j}\left(\sigma_{0}\right)$, there are at least $2^{m}$ extensions of $\tau$ of length $l^{\prime}$ which are compatible with $T_{j}\left(\sigma_{1}\right)$.

Proof. The proof is by induction on $m$. Let $X$ and $l_{0}$ be as in the statement of the lemma. If $m=1$, then let $X^{\prime}=X$ and let $l_{1}=l_{0}$. If $m>1$, then, by the induction hypothesis, we can let $X^{\prime}$ be the set of all strings of some fixed length $l_{1}>l_{0}$ in $\Lambda$ which extend some element of $X$, and we can suppose that for every string $\sigma_{1}$ in $X^{\prime}$ which extends some element $\sigma_{0}$ of $X$, and for each leaf $\tau$ of $T_{j}\left(\sigma_{0}\right)$, there are at least $2^{m-1}$ extensions of $\tau$ of length $l_{1}^{\prime}$ which are compatible with $T_{j}\left(\sigma_{1}\right)$. Now (in both of the cases $m=1$ and $m>1$ ), let $l_{2}^{\prime}$ be the length of the longest string in any $T_{j}(\sigma)$ such that $\sigma \in X^{\prime}$. By compactness there must exist $l_{2}>l_{1}$ such that, for all $\sigma \in \Lambda$ of length $l_{2}, \Psi_{j}\left(\sigma ; l_{2}^{\prime}+1\right) \downarrow$. So far the point is just this: for each $\sigma_{1} \in \Lambda$ of length $l_{2}$ extending some element $\sigma_{0}$ of $X^{\prime}$, and for each leaf $\tau$ of $T_{j}\left(\sigma_{0}\right)$, there are at least 2 incompatible extensions of $\tau$ in $T_{j}\left(\sigma_{1}\right)$. The remaining problem is that these strings may split below many different lengths. So define $l^{\prime}$ to be the length of the longest string in any of the sets $T_{j}(\sigma)$ for $\sigma \in \Lambda$ of length $l_{2}$. Find $l>l_{2}$ such that for every $\sigma \in \Lambda$ of length $l, \Psi_{j}\left(\sigma ; l^{\prime}\right) \downarrow$, the point being that all the strings in $\chi\left(\Psi_{j}\left(\sigma ; l^{\prime}\right)\right)$ must be of length at least $l^{\prime}$. Then $l$ and $l^{\prime}$ satisfy the conditions in the statement of the lemma, as required.

3.3. Dealing with the fact that we do not know the measure. Now we address the first problem raised above; we do not actually get to work within a $\Pi_{1}^{0}$ class on which $\Psi_{j}$ is total. In fact we do not even know the measure of the set on which it is total. Let us suppose for a moment, however, that we are able to find a set of strings $\Pi_{1}$ much as in the simplified example, but which satisfies some further properties:

- There is only a small measure of sets $A$ which do not extend a string in $\Pi_{1}$ and such that $\Psi_{j}(A)$ is total.

- A stronger colouring condition holds. For each element $\sigma$ of $\Pi_{1}$ it is not simply the case that there exist two strings compatible with $T_{j}(\sigma)$ which are given the same colour as $\sigma$. In fact, for some large number $t$, for every $\sigma \in \Pi_{1}$ and every $r<t$, there are at least two strings compatible with $T_{j}(\sigma)$ which are given the 2-coordinate colour $(i, r)$, where $i$ is the colour of $\sigma$.

The rough idea is now as follows. As before we wish to define a computable construction which defines the functionals $\Phi$ and $\Xi$. Given the situation describing $\Pi_{1}$ above, we can now divide the total measure into $t$ pieces. Initially we declare $\Pi_{1}$ to be in state 1 . Rather than simply defining $\Pi_{2}$ in one pass, we shall enumerate strings into it as the construction progresses. As we look to define $\Pi_{2}$, to begin with we look only for a set of strings of measure $1 / t$ on which $\Psi_{j}$ is defined on sufficiently many arguments that we can do the appropriate colouring without losing too much measure. Once such a set of strings is found, we take the set for which the colouring worked, $X_{0}$ say, we enumerate these strings into $\Pi_{2}$ and we declare $\Pi_{1}$ to be in state 2. For the strings $\sigma \in X_{0}$, we define $\Xi(\sigma ; 1)$ by choosing from amongst the strings 
which were given the second colour coordinate 0 when we defined $\Pi_{1}$. So at this point we have enumerated measure close to $1 / t$ into $\Pi_{2}$.

Then we proceed to search for a further measure $1 / t$ on which we can perform a colouring and then enumerate a high proportion of the strings into $\Pi_{2}$ (so this second set of strings $X_{1}$ must all be incompatible with the strings in $X_{0}$ ). If we find this second set of strings $X_{1}$ which we can enumerate into $\Pi_{2}$, then we declare $\Pi_{1}$ to be in state 3 , and for the strings $\sigma \in X_{1}$, we define $\Xi(\sigma ; 1)$ by choosing from amongst the strings which were given the second colour coordinate 1 when we defined $\Pi_{1}$. This means that if $\sigma_{0} \in X_{0}$ and $\sigma_{1} \in X_{1}$, then all strings in $\Xi\left(\sigma_{0} ; 1\right)$ are incompatible with the strings in $\Xi\left(\sigma_{1} ; 1\right)$. We then proceed to search for another set of strings $X_{3}$, and so on. Meanwhile, of course, we also begin to enumerate strings into $\Pi_{3}$, but at this level we divide the measure into $t^{\prime}$ pieces for some $t^{\prime}>t$.

Now let us see roughly why this works. Recall that our task is to define the set $W_{f(j, n)}^{\emptyset^{\prime}}$ as in the statement of Lemma 3.1. As we proceed according to the process described above, there are two reasons that we have to enumerate strings into this set. First of all, the set $\Pi_{1}$ was chosen so that there exists a small measure of strings $A$ for which $\Psi_{j}(A)$ is total but such that $A$ does not extend a string in $\Pi_{1}$. We shall cover this set with a set of small measure $V_{0}$. Each time we perform a colouring and try to add more strings into $\Pi_{2}$, the colouring only works for a certain large proportion of the strings concerned. We do not immediately have to enumerate the strings for which the colouring does not work into $W_{f(j, n)}^{\emptyset^{\prime}}$, however, since these strings still have another chance to be enumerated into $\Pi_{2}$ when we search for the next measure $1 / t$ on which to perform a colouring (and a similar analysis applies, of course, as we go to enumerate strings into $\Pi_{x}$ for $x>2$ ). When $\Pi_{1}$ reaches its final state $(\leq t+1)$, however, since we always require measure at least $1 / t$ on which to perform our next colouring, we may be left with a set of strings $A$ of measure $<1 / t$ for which $\Psi_{j}(A)$ is total, but for which we do not define $\Xi(A ; 1)$ (and of course this will also happen for $\Pi_{x}$ with $x>1$ and $t^{\prime}>t$ ). These strings we enumerate into a set $V_{1}$ which will be c.e. relative to $\emptyset^{\prime}$. Thus, ultimately we shall define

$$
W_{f(j, n)}^{\emptyset^{\prime}}=V_{0} \cup V_{1}
$$

and we shall ensure that each $V_{i}$ is of appropriately small measure.

3.4. Producing the correct combinatorial lemma. In light of the previous discussion, we need a modified version of Lemma 3.3 .

Lemma 3.5. Suppose that $X$ and $Y$ are finite and non-empty and that for each $y<2^{m_{1}}$ and for each $a \in X, f_{y}(a)$ is a subset of $Y$ of size $2^{m_{2}}$. If each element of $Y$ is coloured with a number $r<t$, consider the proportion of the $a \in X$ for which the following condition holds: for every $y<2^{m_{1}}$ and every $r<t$ there exist at least $2^{d}$ elements of $f_{y}(a)$ which are given colour $r$. For fixed values of $m_{1}, d, k \in \omega$ and $t \geq 1$, if $m_{2}$ is chosen sufficiently large, then we may colour $Y$ so that this proportion is at least $1-2^{-k}$. In fact, there exists a computable function $\pi_{1}$, such that if $m_{2} \geq \pi_{1}\left(m_{1}, k, d, t\right)$, then this condition will be satisfied.

Proof. The proof is by induction on $t$. The case $t=1$ is trivial, so we begin by considering the case $t=2$. The proof in this case is almost identical to the proof of Lemma 3.3. We just make the following minor changes. First we form a modified version of the 'standard method' from the proof of Lemma 3.2. At stage $s+1$ we say that giving $b \in Y_{s}$ colour $i$ aids $a \in X_{s}$ if $a$ is associated with $b$ and there is already 
some $b^{\prime} \in Y$ which is associated with $a$ and which has been given colour $1-i$. We say that giving $b \in Y_{s}$ colour $i$ hinders $a \in X_{s}$ if $a$ is associated with $b$ and there is already some $b^{\prime} \in Y$ which is associated with $a$ and which has been given colour $i$. Note that if giving $b$ colour $i$ hinders $a$, then giving it colour $1-i$ aids $a$, so when we go to colour $b$ it is still always possible to choose a colour which aids at least as many $a \in X_{s}$ as it hinders. We define $X_{s+1}$ to be the set of $a \in X_{s}$ for which it is not yet the case that for each $r<2$ there is some element of $Y$ associated with $a$ which has been coloured $r$. The only remaining change is that then, after forming the set $X^{\prime}$ just as before, we use the modified version of the standard method for colouring $X^{\prime}$ and $Y$, and we regard the colouring as being good for $c \in X^{\prime}$ if for each $r<2$ there is some element of $Y$ associated with $c$ which has been coloured $r$. Now the total number of elements $c$ of $X^{\prime}$ such that the colouring is not good for $c$ is at most $\frac{2^{m_{1}+d}|X|}{2^{m_{2}-d}}$, and we are able to conclude that the proportion of $a \in X$ for which the colouring is good is at least $1-\frac{2^{m_{1}+d}}{2^{m_{2}-d}}$.

In order to prove the induction step, suppose the result holds for $t$. Fix $m_{1}, d$ and $k$. Let $m_{2}>d$ be large enough so that if each element of $X$ is associated with $2^{m_{2}}$ elements of $Y$, then we may colour each element of $Y$ with a number $<t$ so that the proportion of $a \in X$ for which the following condition holds is at least $1-2^{-(k+1)}$; for every $y<2^{m_{1}}$ and every $r<t$ there exist at least $2^{d}$ elements of $f_{y}(a)$ which are given colour $r$. Then let $m_{3}$ be sufficiently large so that if each element of $X$ is associated with $2^{m_{3}}$ elements of $Y$, then we may colour each element of $Y$ with a number $<2$ so that the proportion of $a \in X$ for which it is the case that for every $y<2^{m_{1}}$ and every $r<2$ there exist at least $2^{m_{2}}$ elements of $f_{y}(a)$ which are given colour $r$, is at least $1-2^{-(k+1)}$.

Now, given any $X$ and $Y$ such that, for each $y<2^{m_{1}}$ and for each $a \in X, f_{y}(a)$ is a subset of $Y$ of size $2^{m_{3}}$, colour each element of $Y$ with a colour $<2$ so that if $X^{\prime}$ is the set of $a \in X$ for which it is the case that for every $y<2^{m_{1}}$ and every $r<2$ there exist at least $2^{m_{2}}$ elements of $f_{y}(a)$ which are given colour $r$, then $\left|X^{\prime}\right| /|X|>1-2^{-(k+1)}$. Then remove the colour from each element of $Y$ which is coloured 1 and change the colour of each element of $Y$ which is coloured 0 to $t$. If we let $Y^{\prime}$ be those elements of $Y$ which now do not have a colour, then for each $a \in X^{\prime}$ and each $y<2^{m_{1}}$, there are at least $2^{m_{2}}$ elements of $f_{y}(a)$ which are in $Y^{\prime}$, so let $f_{y}^{\prime}(a)=f_{y}(a) \cap Y^{\prime}$. According to the choice of $m_{2}$, we may now colour the elements of $Y^{\prime}$ so that the proportion of $a \in X^{\prime}$ for which it is the case that for every $y<2^{m_{1}}$ and every $r<t$ there exist at least $2^{d}$ elements of $f_{y}^{\prime}(a)$ which are given colour $r$ is at least $1-2^{-(k+1)}$. Therefore the total proportion of $a \in X$ for which it is the case that for every $y<2^{m_{1}}$ and every $r<t+1$ there exist at least $2^{d}$ elements of $f_{y}(a)$ which are given colour $r$ is at least $\left(1-2^{-(k+1)}\right)\left(1-2^{-(k+1)}\right)>1-2^{-k}$, as required.

Finally, the following is the lemma that we actually need.

Lemma 3.6. Let $X$ and $Y$ be non-empty finite sets. Suppose that for each $y<2^{m_{1}}$ and for each $a \in X, f_{y}(a)$ is a subset of $Y$ of size $2^{m_{2}}$. Let each element of $X$ be given a colour, either 0 or 1 . If each element of $Y$ is given a 2-coordinate colour $(i, r)$ such that $i<2$ and $r<t$, then consider the proportion of $a \in X$ for which the following condition holds: for every $y<2^{m_{1}}$ and every $r<t$ there exist at least two elements of $f_{y}(a)$ which are given colour $(i, r)$, where $i$ is the colour of $a$. For fixed values of $m_{1}, k \in \omega$ and $t \geq 1$, if $m_{2}$ is chosen sufficiently large, then 
we may colour $Y$ so that this proportion is at least $1-2^{-k}$. In fact, there exists a computable function $\pi$ such that if $m_{2} \geq \pi\left(m_{1}, k, t\right)$, then this condition will be satisfied.

Proof. This follows almost directly from Lemma 3.5. Since it is required that there should be two elements of $f_{y}(a)$ which are given colour $(i, r)$, rather than giving 2 -coordinate colours we can consider giving 3 -coordinate colours $\left(i^{\prime}, i, r\right)$ for $i, i^{\prime}<2$ and $r<t$. For each $a \in X$ and each $y<2^{m_{1}}$ we then hope that, for every $i, i^{\prime}<2$ and every $r<t$ there is some element of $f_{y}(a)$ which is given the colour $\left(i^{\prime}, i, r\right)$. That we can achieve this for a sufficiently high proportion of the $a \in X$ follows from Lemma 3.5 simply by coding 3-coordinate colours as standard colours of a single coordinate.

3.5. Three measure-theoretic lemmas. Now that we have the correct combinatorial lemma in place, we have to be able to translate this into a measure-theoretic statement.

The next lemma may initially sound a little technical, but it is really just a simple translation of Lemma 3.4 into a statement about measure. The set $Z$ in the statement of the lemma does not really have much impact on its proof, and the reader may find it helpful to omit all references to $Z$ on a first reading.

Lemma 3.7. Let $X, Z \subseteq 2^{<\omega}$ be finite and prefix-free sets of strings such that every element of $Z$ extends an element of $X$. Given $k \in \omega$, consider the class $P$ of sets $A$ which extend an element of $X$ and do not extend any element of $Z$, and for which $\Psi_{j}(A)$ is total. If $\mu(P)>2^{-k}$, then for any $m$, there exists a finite prefix-free set $X^{\prime}$ such that all strings in $X^{\prime}$ extend an element of $X$ and are incompatible with all strings in $Z$, such that $\mu\left(X^{\prime}\right) \geq 2^{-k}$, and which satisfies the following condition. There exists $l$ such that, for all strings $\sigma_{1} \in X^{\prime}$ which extend a string $\sigma_{0} \in X$, and for each leaf $\tau$ of $T_{j}\left(\sigma_{0}\right)$, there are at least $2^{m}$ strings extending $\tau$ of length $l$ which are compatible with $T_{j}\left(\sigma_{1}\right)$.

Proof. Suppose that $P$ is of measure $2^{-k}+\epsilon$ for $\epsilon>0$. Take an open cover $Q$ of the class of sets $A$ for which $\Psi_{j}(A)$ is partial and such that the measure of all $A \in Q$ such that $\Psi_{j}(A)$ is total is less than $\epsilon$. Then the complement of $Q$ intersected with the set of all sets which extend an element of $X$ but do not extend any element of $Z$ is a closed set of measure $>2^{-k}$. The result then follows directly from Lemma 3.4

Now we wish to combine Lemma 3.7 with Lemma 3.6. Let $X, Z \subseteq 2^{<\omega}$ be finite and prefix-free sets of strings such that every element of $Z$ extends an element of $X$. Roughly speaking, by an extension set for the pair $(X, Z)$, we mean a set of strings which extend strings in $X$ and are incompatible with all elements of $Z$, which is of 'sufficiently' large measure and on which we can perform a successful colouring.

Now let us make this precise. Suppose that we are given $k_{0}, k_{1}, k_{2} \in \omega$ such that $k_{1}>k_{0}$, and that $X^{\prime}$ is a finite set of strings such that every element of $X^{\prime}$ extends an element of $X$ and is incompatible with all elements of $Z$. Suppose further that we assign the colour either 0 or 1 to each element of $X^{\prime}$. For any $l$ and any assignment of 2-coordinate colours $(i, r)$ to all strings of length $l$, we may then consider the proportion of $\sigma_{1} \in X^{\prime}$ for which the following holds: if $\sigma_{1} \supset \sigma_{0} \in X$, then for each leaf $\tau$ of $T_{j}\left(\sigma_{0}\right)$ and for every $r<k_{2}$ there exist at least two strings extending $\tau$ of length $l$ which are compatible with $T_{j}\left(\sigma_{1}\right)$ and which are given colour $(i, r)$, where $i$ 
is the colour of $\sigma_{1}$. If there exists $l$ such that no matter how we assigned the colours to elements of $X^{\prime}$, the strings of length $l$ can be coloured so that this proportion is greater than $1-2^{-k_{1}}$, and if $\mu\left(X^{\prime}\right) \geq 2^{-k_{0}}-2^{-k_{1}}$, then we say that $X^{\prime}$ is an extension set for the pair $(X, Z)$ and the triple $\left(k_{0}, k_{1}, k_{2}\right)$. We also say that $l$ is an appropriate axiom length for this extension set.

Lemma 3.8. Let $X, Z \subseteq 2^{<\omega}$ be finite and prefix-free sets of strings such that every element of $Z$ extends an element of $X$. Suppose we are given $k_{0}, k_{1}, k_{2} \in \omega$ such that $k_{1}>k_{0}$, and consider the class $P$ of sets $A$ which extend an element of $X$ and do not extend any element of $Z$, and for which $\Psi_{j}(A)$ is total. If $\mu(P) \geq 2^{-k_{0}}$, then there exists $X^{\prime}$ which is an extension set for $(X, Z)$ and $\left(k_{0}, k_{1}, k_{2}\right)$.

Proof. This follows directly from Lemma 3.7 and Lemma 3.6

We also need a lemma which allows us to define $\Pi_{1}$.

Lemma 3.9. There exists $g \leq_{T} \emptyset^{\prime}$ which, given input $\left(j, k_{1}, k_{2}\right)$, produces a prefixfree and finite set of strings $X$ and a pair $(m, l)$ such that:

(1) for every string $\sigma \in X$ there exist at least $2^{k_{2}}$ many strings of length $l$ which are compatible with $T_{j}(\sigma)$;

(2) if $P$ is the set of strings $A$ which do not extend any string in $X$ and such that $\Psi_{j}(A ; m) \downarrow$, then $\mu(P)<2^{-k_{1}}$.

Proof. Given $j, k_{1}, k_{2}$, it suffices to show that $X, m$ and $l$ as in the statement of the lemma exist, since then an oracle for $\emptyset^{\prime}$ can certainly verify that they satisfy the required properties (i.e. the set of valid outputs is c.e. in $\left.\emptyset^{\prime}\right)$. Let $Q$ contain precisely those sets on which $\Psi_{j}$ is partial, and suppose $\mu(Q)=\epsilon$. Let $U$ be an open covering of $Q$ such that $\mu(U)<\epsilon+2^{-k_{1}}$. Then the measure of the sets in $U$ for which $\Psi_{j}$ is total is less than $2^{-k_{1}}$, so there exists $m$ such that the measure of the sets $A$ in $U$ for which $\Psi_{j}(A ; m) \downarrow$ is less than $2^{-k_{1}}$. The complement of $U$ is a closed set, so the result follows from Lemma 3.4 .

3.6. Construction of $f, \Xi, \Phi$ on input $j, n$. Now we are ready to start defining the function $f$ as in the statement of Lemma 3.1 So suppose we are given inputs $j$ and $n$. We let $\kappa$ be a computable function such that $\sum_{x \geq 0} 2^{-\kappa(x)}<2^{-n}$.

As stated previously, we shall run a computable construction which will define $\Xi$ and $\Phi$ and which will enumerate all the sets $\Pi_{x}$ for $x>1$, but first we have to make one use of the function $g$ provided by Lemma 3.9 in order to help us define $\Pi_{1}$. Given $\pi$ as in the statement of Lemma 3.6. we let $m=\pi\left(0, \kappa(0)+1,2^{\kappa(1)}\right)$ and then we let $X^{*}$ and $\left(m^{*}, l^{*}\right)$ be the outputs of $g$ given the input $(j, \kappa(0)+1, m)$.

As promised, the set $W_{f(j, n)}^{\emptyset^{\prime}}$ will actually be constructed as the union of two sets, $V_{0}$ and $V_{1}$. At this point we enumerate into $V_{0}$ all $\sigma$ which do not extend any element of $X^{*}$ and such that $\Psi_{j}\left(\sigma ; m^{*}\right) \downarrow$. So far then, we have enumerated measure $<2^{-(\kappa(0)+1)}$ into this set.

Our next task is to define the functionals $\Xi$ and $\Phi$. At each stage of the construction, $\Pi_{x}$ will be regarded as having a state $d \in \omega$. Initially $\Pi_{x}$ is empty and is in state 1 , and its state may be increased by 1 a finite number of times. At stage $s$, we say that $\Pi_{x}$ requires attention if it is non-empty and (letting $\Pi_{x}, \Pi_{x+1}$ and $d$ take their present values) there exists an extension set $X^{\prime}$ for the pair $\left(\Pi_{x}, \Pi_{x+1}\right)$ and the triple $\left(\kappa(x), \kappa(x)+d+1,2^{\kappa(x+1)}\right)$ with all strings in $X^{\prime}$ of length $s$. 
During the construction, it is also helpful to enumerate some auxiliary sets $S_{x}(\sigma)$ and $S(\sigma)$. The set $S_{x}(\sigma)$ is simply a set of strings which may be enumerated into $\chi\left(\Xi\left(\sigma^{\prime}\right)\right)$ for $\sigma^{\prime} \supset \sigma$ and $S_{x}$ is just the union of all $S_{x}(\sigma)$ such that $\sigma \in \Pi_{x}$.

3.6.1. The computable construction of $\Xi$ and $\Phi$. At each stage $s$ we proceed as follows.

Stage $s=0$. We define $\Xi(\lambda ; 0)=\chi^{-1}(\{\lambda\})$.

Stage $s=1$. Let $X^{*}$ and $l^{*}$ be as defined above. If $X^{*}$ is non-empty, then perform the following instructions:

(1) Give the strings of length $l^{*}$ each a colour $(i, r)$ so that the proportion of those strings $\sigma$ in $X^{*}$ which satisfy the following condition is greater than $1-2^{-(\kappa(0)+1)}$ : for every $r<2^{\kappa(1)}$ there are at least two strings $\tau$ of length $l^{*}$ which are compatible with $T_{j}(\sigma)$ and which are given colour $(\sigma(0), r)$. Enumerate all $\sigma \in X^{*}$ for which the latter condition is not satisfied into $V_{0}$ (so we enumerate in a set of strings of measure at most $2^{-(\kappa(0)+1)}$ ).

(2) For all strings $\sigma$ in $X^{*}-V_{0}$, and for each $r<2^{\kappa(1)}$, take two strings of length $l^{*}$ which are compatible with $T_{j}(\sigma)$ and have been given colour $(\sigma(0), r)$ and enumerate these into $S_{1}(\sigma)$ and $S_{1}$.

(3) For each string $\tau$ which we have just coloured $(i, r)$ enumerate the axiom $\Phi(\tau ; 0)=i$.

(4) Enumerate all strings in $X^{*}-V_{0}$ into $\Pi_{1}$.

Stage $s>1$. Check to see whether there exists any $\Pi_{x}$ which requires attention. If not, then proceed to stage $s+1$, otherwise let $x$ be the least such, and suppose that $\Pi_{x}$ is in state $d$. We say that $\Pi_{x}$ receives attention. Let $X^{\prime}$ be an extension set for the pair $\left(\Pi_{x}, \Pi_{x+1}\right)$ and the triple $\left(\kappa(x), \kappa(x)+d+1,2^{\kappa(x+1)}\right)$, such that all strings in $X^{\prime}$ are of length $s$. Let $l$ be an appropriate axiom length for this extension set. Perform the following instructions:

(1) For each $\sigma_{1} \in X^{\prime}$, suppose that $\sigma_{1} \supset \sigma_{0} \in \Pi_{x}$. We shall establish in the verification that $\Pi_{x}$ never requires attention after entering state $2^{\kappa(x)}+1$, so for each string $\tau$ in $\Xi\left(\sigma_{0} ; x-1\right)$, there are precisely two strings $\tau^{\prime}$ in $S_{x}\left(\sigma_{0}\right)$ which have colour $\left(\sigma_{0}(x-1), d-1\right)$. Define $\Xi\left(\sigma_{1} ; x\right)$ to be the code for this set of $2^{x}$ many strings $\tau^{\prime}$ as we vary $\tau$ over the strings in $\Xi\left(\sigma_{0} ; x-1\right)$.

(2) Now consider $V$ which is the set of all strings $\tau$ of length $l$ for which it is the case that there is some string $\sigma_{1} \in X^{\prime}$ and some $\tau^{\prime} \in \Xi\left(\sigma_{1} ; x\right)$, such that $\tau$ extends $\tau^{\prime}$. Give each string in $V$ a colour $(i, r)$ such that the proportion of the strings $\sigma_{1} \in X^{\prime}$ for which the following condition is satisfied is greater than $1-2^{-(\kappa(x)+d+1)}$ : for each string $\tau^{\prime} \in \Xi\left(\sigma_{1} ; x\right)$ and for each $r<2^{\kappa(x+1)}$, there are at least two strings in $V$ compatible with $T_{j}\left(\sigma_{1}\right)$ which are given colour $\left(\sigma_{1}(x), r\right)$. Enumerate all strings in $X^{\prime}$ for which the latter condition is satisfied into $\Pi_{x+1}$.

(3) For all strings $\sigma_{1} \in X^{\prime}$ which we have just enumerated into $\Pi_{x+1}$, for each string $\tau^{\prime} \in \Xi\left(\sigma_{1} ; x\right)$ and for each $r<2^{\kappa(x+1)}$, take two strings in $V$ extending $\tau^{\prime}$ which are compatible with $T_{j}\left(\sigma_{1}\right)$ and have been given colour $\left(\sigma_{1}(x), r\right)$ and enumerate these into $S_{x+1}\left(\sigma_{1}\right)$ and $S_{x+1}$.

(4) For each string $\tau$ which we have just coloured $(i, r)$ enumerate the axiom $\Phi(\tau ; x)=i$.

(5) Declare that $\Pi_{x}$ is now in state $d+1$. 
3.6.2. Definition of $V_{1}$. For each $x$, let $\Pi_{x}$ take its final value. For all $m, x \in \omega$, let $V(m, x)$ be the set of all strings $\sigma$ which extend an element of $\Pi_{x}$, which are incompatible with any strings in $\Pi_{x+1}$ and such that $\Psi_{j}(\sigma ; m) \downarrow$. If $\mu(V(m, x))<$ $2^{-\kappa(x)}$, then enumerate all strings in $V(m, x)$ into $V_{1}$. We say that these strings are enumerated into $V_{1}$ for the sake of $x$.

3.7. Verification of $f, \Xi, \Phi$ on input $j, n$. We must prove that the measure of $W_{f(j, n)}^{\emptyset^{\prime}}$ is less than $2^{-n}$ and that the axioms we enumerate for $\Phi$ are consistent. In order to prove that the construction is well defined we must also show that $\Pi_{x}$ never requires attention when in state $2^{\kappa(x)}+1$. Finally, we must also prove that if $\Psi_{j}(A)$ is total but $\Xi(A)$ is partial, then $A \in\left[\left[W_{f(j, n)}^{\emptyset^{\prime}}\right]\right]$.

Lemma 3.10. The measure of $W_{f(j, n)}^{\emptyset^{\prime}}$ is less than $2^{-n}$.

Proof. We have that $W_{f(j, n)}^{\emptyset^{\prime}}=V_{0} \cup V_{1}$. There are two points at which we enumerate strings into $V_{0}$, once prior to the computable construction of $\Xi$ and $\Phi$, and once at stage 1 . We have that $\mu\left(V_{0}\right)<2^{-\kappa(0)}$. Next consider $V_{1}$. Fix $x$ and let $\Pi_{x}$ and $\Pi_{x+1}$ take their final values. If there does not exist any $m$ such that $\mu(V(m, x))<$ $2^{-\kappa(x)}$, then we do not enumerate any strings into $V_{1}$ for the sake of $x$. Otherwise there exists a least $m$ for which this condition holds, $m_{0}$ say. According to the conventions described in Section 2, if $m>m_{0}$, then for any $\sigma, \Psi_{j}(\sigma ; m) \downarrow$ implies $\Psi_{j}\left(\sigma, m_{0}\right) \downarrow$. So $V(m, x) \subseteq V\left(m_{0}, x\right)$ for all $m \geq m_{0}$, and the measure of the set of strings enumerated into $V_{1}$ for the sake of $x$ is less than $2^{-\kappa(x)}$. Thus $\mu\left(V_{1}\right)<$ $\sum_{x>0} 2^{-\kappa(x)}$.

Lemma 3.11. The axioms enumerated for $\Phi$ are consistent.

Proof. It is clear that the axioms enumerated at a single stage of the construction are consistent with each other, and the axioms enumerated when $\Pi_{x}$ receives attention are on argument $x$, so if two axioms $\Phi(\tau ; x)=i$ and $\Phi\left(\tau^{\prime} ; x^{\prime}\right)=1-i$ are to be inconsistent with each other, it must be that $x=x^{\prime}$, that the first is enumerated when $\Pi_{x}$ receives attention and is in state $d$, and the second is enumerated when $\Pi_{x}$ receives attention and is in state $d^{\prime} \neq d$. Thus there is a least $y \leq x$ such that $\tau$ and $\tau^{\prime}$ have initial segments enumerated into $S_{y+1}$ while $\Pi_{y}$ is in different states, say $d_{0}$ and $d_{1}$ respectively. Then $\tau$ and $\tau^{\prime}$ extend strings $\tau_{0}$ and $\tau_{1}$ respectively, which were enumerated into $S_{y}$ at the same stage and given colours $\left(i, d_{0}-1\right)$ and $\left(i^{\prime}, d_{1}-1\right)$ for some $i, i^{\prime}$, and so are incompatible.

It is clear that if $\Xi(A)$ is total, then it is a perfect tree $T^{\prime}$ such that every path $B$ through $T^{\prime}$ is also a path through $T$ which is computed by $A$ via $\Psi_{j}$, with $\Phi(B)=A$. It just remains to prove our last two lemmas.

Lemma 3.12. $\Pi_{x}$ never requires attention after entering state $2^{\kappa(x)}+1$.

Proof. When $\Pi_{x}$ receives attention and is in state $d$, we enumerate a set of strings of measure at least $\left(1-2^{-(\kappa(x)+d+1)}\right)\left(2^{-\kappa(x)}-2^{-(\kappa(x)+d+1)}\right) \geq 2^{-\kappa(x)}-2^{-(\kappa(x)+d)}$ into $\Pi_{x+1}$ (consider the measure of the extension set and the proportion of strings for which the colouring works). If we enumerate $\sigma$ and $\sigma^{\prime}$ into $\Pi_{x+1}$ at different stages $s$ and $s^{\prime}$ when $\Pi_{x}$ receives attention, then $\sigma$ and $\sigma^{\prime}$ are incompatible. So if $\Pi_{x}$ was to be in state $2^{\kappa(x)}+1$ and require attention, then the measure of the relevant extension set union $\Pi_{x+1}$ would be strictly greater than 1 , a contradiction. 
Lemma 3.13. If $\Psi_{j}(A)$ is total and $\Xi(A)$ is partial, then $A$ extends some string in $V_{0}$ or $V_{1}$.

Proof. Suppose that $\Psi_{j}(A)$ is total and $\Xi(A)$ is partial and that $A$ does not extend any string in $V_{0}$. We must show that it extends a string in $V_{1}$. So let $x$ be the greatest number such that an initial segment of $A$ is enumerated into $\Pi_{x}$. Let $d$ be the greatest state that $\Pi_{x}$ is in at any stage of the construction, and let $\Pi_{x}$ and $\Pi_{x+1}$ take their final values. Let $V$ be the set of all $B$ extending a string in $\Pi_{x}$, such that $B$ is incompatible with any string in the final value $\Pi_{x+1}$ and such that $\Psi(B)$ is total. By Lemma 3.8, $\mu(V)<2^{-\kappa(x)}$, since otherwise there would be a stage at which $\Pi_{x}$ receives attention and enters state $d+1$. Therefore there exists $m$ such that $\mu(V(m, x))<2^{-\kappa(x)}$. All strings in $V(m, x)$ are enumerated into $V_{1}$, and $A$ extends some string in this set, as required.

This concludes the proof of Lemma 3.1] which in turn implies Theorem 1.1

\section{REFERENCES}

[BDN11] George Barmpalias, Rod Downey, and Keng-Meng Ng. Jump inversions inside effectively closed sets and applications to randomness. J. Symbolic Logic, 2011. In press.

[Coo04] S. Barry Cooper. Computability Theory. CRC Mathematics Series. Chapman \& Hall, Boca Raton, FL, New York, London, 2004. MR2017461 (2005h:03001)

[DH10] Rod Downey and Denis Hirschfeldt. Algorithmic Randomness and Complexity. SpringerVerlag, 2010. MR2732288

[DJS96] Rod Downey, Carl G. Jockusch, Jr., and Michael Stob. Array nonrecursive sets and genericity. In Computability, Enumerability, Unsolvability: Directions in Recursion Theory, volume 224 of London Mathematical Society Lecture Notes Series, pages 93104. Cambridge University Press, 1996. MR 1395876 (97f:03060)

[Joc80] C. Jockusch, Jr. Degrees of generic sets. In F. R. Drake and S. S. Wainer, editors, Recursion Theory: Its Generalizations and Applications, Proceedings of Logic Colloquium '79, Leeds, August 1979, pages 110-139, Cambridge University Press, Cambridge, U. K., 1980. MR598304 (83i:03070)

[Kum00] Masahiro Kumabe. A 1-generic degree with a strong minimal cover. J. Symbolic Logic, 65(3):1395-1442, 2000. MR1791382 (2001m:03080)

[Lew07a] Andrew E. M. Lewis. $\Pi_{1}^{0}$ classes, strong minimal covers and hyperimmune-free degrees. Bull. Lond. Math. Soc., 39(6):892-910, 2007. MR2392813 (2009b:03113)

[Lew07b] Andrew E. M. Lewis. A random degree with strong minimal cover. Bull. Lond. Math. Soc., 39(5):848-856, 2007. MR2365234 (2008i:03049)

[Lew09] Andrew E. M. Lewis. Strong minimal covers and a question of Yates: the story so far. In Logic Colloquium 2006, Lect. Notes Log., pages 213-228. Assoc. Symbol. Logic, Chicago, IL, 2009. MR2562554 (2010j:03039)

[Mar0s] D. Martin. Measure, category, and degrees of unsolvability. Unpublished manuscript, 1960s.

[ML66] Per Martin-Löf. The definition of random sequences. Information and Control, 9:602619, 1966. MR0223179(36:6228)

[Nie09] André Nies. Computability and Randomness. Oxford University Press, 2009. MR2548883

[Sac63] G. E. Sacks. Degrees of Unsolvability, volume 55 of Annals of Mathematical Studies. Princeton University Press, 1963. MR 0186554 (32:4013)

[Soa87] Robert I. Soare. Recursively enumerable sets and degrees. Perspectives in Mathematical Logic. Springer-Verlag, Berlin, 1987. A study of computable functions and computably generated sets. MR882921 (88m:03003)

[Spe56] C. Spector. On the degrees of recursive unsolvability. Ann. of Math. (2), 64:581-592, 1956. MR0082457(18:552d) 
Institute for Logic, Language and Computation, Universiteit van Amsterdam 1090 GE, P.O. Box 94242, The Netherlands

E-mail address: barmpalias@gmail.com

$U R L:$ http://www.barmpalias.net

School of Mathematics, University of Leeds, LS2 9JT Leeds, United Kingdom

E-mail address: andy@aemlewis.com

URL: http://www. aemlewis.com 